\section{QUANTUM CASCADE LASERS}

\section{On-chip dual-comb source}

Appl. Phys Lett. 108, 171104 (2016)

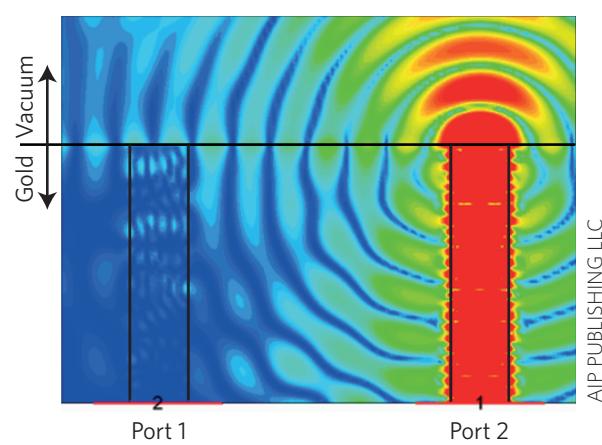

Dual-comb spectroscopy based on terahertz $(\mathrm{THz})$ quantum cascade lasers (QCLs) can be a powerful tool for molecular fingerprint characterization. However, sensitive, fast detectors in the $\mathrm{THz}$ frequency range are usually required. Now, Markus Rösch and colleagues from ETH Zurich have developed an on-chip $\mathrm{THz}$ dual-comb set-up that does not require a fast detector. Two THz QCLs were processed next to each other by dry-etching techniques on the same chip and connected individually to two current sources. The two lasers had the same width $(50 \mu \mathrm{m})$ and cavity length $(1.9 \mathrm{~mm})$, and were spaced $450 \mu \mathrm{m}$ apart. A small amount of light from one laser perturbed the other laser. The dynamics of the second laser thus act as an efficient ultrafast detector. A multi-heterodyne spectrum was detected for up to 30 modes corresponding to a total bandwidth of $630 \mathrm{GHz}$. The Swiss scientists further used their device to measure the spacing of the modes of the comb, showing that they are equally spaced to an accuracy of $10^{-12}$ at a frequency of $2.5 \mathrm{THz}$.

\section{NANOPHOTONICS}

\section{Giant nonlinearity \\ Science 352, 795-797 (2016)}

Any material with free charges described by the Drude model, including metals or sufficiently doped semiconductors, can exhibit permittivity near zero at the transition frequency between metal and dielectric behaviour. Applications for the epsilon-nearzero regime may include subwavelength waveguiding, enhanced third-harmonic generation and ultrafast optical switching. Now a team of researchers from Canada, Mexico and the US have observed an optical nonlinear response from indium tin oxide at epsilon-near-zero frequencies that is two orders of magnitude larger than for $\mathrm{As}_{2} \mathrm{Se}_{3}$ chalcogenide glass. The team achieved a $170 \%$ variation in the real part of the refractive index, due to a change in permittivity from $0+0.352 i$ to $1.22+0.61 i$, and propose that the strong response is related to laser-induced electron 'heating' and modification of the energy distribution of the conduction band. In their experiment, the authors used commercially available indium tin oxide with an epsilonnear-zero wavelength of 1,240 nm. Near this wavelength they find a nonlinear index coefficient $\sim 43$ times higher than at $970 \mathrm{~nm}$. For transverse magnetic-polarized light and optimal angles of incidence, the nonlinear coefficient is even larger. The material recovers from the pulses on a timescale of just $360 \mathrm{fs}$, which the authors point out may enable modulation up to $1.5 \mathrm{THz}$.

\section{METAMATERIALS}

\section{Ultracold atom lattices}

Phys. Rev. Lett. 116, 165502 (2016)

Although research in metamaterials has witnessed a rapid growth in recent years, the presence of large optical losses

IMAGING

The realization of an imaging technique that can track multiple synaptic proteins in neuronal cells would potentially deliver precious information on the effects of neurological and psychiatric treatments. Hyperspectral imaging gives access to many narrow spectral bands over a continuous frequency range, thus avoiding spectral overlaps that limit the number of neuroreceptors observed with conventional techniques. Following this research direction, Simon Labrecque and co-workers in Canada have designed a hyperspectral microscope that can optically track distinct synaptic proteins in a live neuron. The core element of the imaging system is a tunable Bragg filter for wavelength selection. The authors used their microscope to acquire a $500 \mathrm{~nm}$ to $850 \mathrm{~nm}$ spectrum in $3.4 \mathrm{~s}$ using a $10-\mathrm{nm}$ step size and 50 -ms exposure time for each measurement, allowing the trajectories of 4 membrane neuroreceptors labelled with different quantum dots to be imaged simultaneously. The authors note that the scheme makes it possible to study the impact of specific substances on receptor mobility. and structural imperfections are still unsolved issues. In an attempt to address such pressing challenges, Pankaj Jha and co-workers in the US and Saudi Arabia have turned to cold atoms in optical lattices to design a new architecture for metamaterials. In their proposed atomic lattice quantum metamaterial, off-resonant and blue-detuned laser beams trap ultracold atoms exhibiting an optical response that can be tuned thanks to two coherent drive fields. The authors theoretically demonstrate that they can engineer a system where the permittivity near an atomic resonance is negative at the lattice sites, and the vacuum between adjacent sites acts as a dielectric medium. Careful manipulation of the parameters that characterize the driving lasers is shown to allow for the complete cancellation of optical absorption, whereas turning off the external coherent fields produces non-zero values for the imaginary part of the permittivity. Further, Jha and collaborators highlight how their scheme can lead to optical lattice metamaterials with hyperbolic dispersion. They predict that, in principle, the approach can be extended to $2 \mathrm{D}$ and $3 \mathrm{D}$ lattices.

NANOPARTICLES

\section{Scattering-force scheme} APL Photon. 1, 026102 (2016)

The scattering force imparted by green light to a single gold nanoparticle in solution has been measured with femtonewton resolution by a German-Danish team of scientists. The researchers used light from a 1,064-nm infrared laser to trap a single 80-nm-diameter gold nanoparticle in water. They then laterally injected, via an optical fibre, intensity-modulated green $(532 \mathrm{~nm}$ ) laser light (resonant to the particle's plasmon frequency) to perturb the motion of the trapped particle in a periodic manner. A video of the nanoparticle's harmonic motion was recorded by a high-speed digital camera operating at a data acquisition speed of $500 \mathrm{~Hz}$ and a frame exposure time of $1.9 \mathrm{~ms}$. Position-tracking software was then used to analyse the nanoparticle's motion and deduce the magnitude of the scattering force, which was determined to be a maximum of $21.1 \mathrm{fN}$ with an uncertainity of $\pm 5.8 \mathrm{fN}$. The high sensitivity of the technique is expected to be valuable for further studies of optomechanics and nanostructure manipulation.

Written by Gaia Donati, Noriaki Horiuchi, David Pile and Oliver Graydon. 